\title{
Transanal TAMIS total mesorectal excision (TME)—a work in progress
}

\author{
S. D. Wexner • M. Berho
}

Received: 24 January 2014/ Accepted: 15 March 2014/Published online: 29 March 2014

(C) Springer-Verlag Italia 2014

Since Heald's [1] landmark publication in The Lancet in 1982, total mesorectal excision (TME) has become the only globally acceptable surgical method for curative rectal extirpation for rectal adenocarcinoma. The advent of TME and the worldwide adoption of this technique has accounted for perhaps the largest and most important advance in our quest to improve cancer care within the specialty of colorectal surgery. During the last 50 years, there have been two other advances; firstly, Norman Nigro [2] discovered that chemoradiotherapy could replace rather than be used prior to abdominoperineal resection for anal carcinoma. More recently, Angelita Habr-Gama [3] demonstrated that this Nigro-type protocol may be applicable in selected patients with rectal carcinoma. However, given the prevalence of rectal carcinoma as compared to anal carcinoma, the advance championed by Bill Heald has clearly had the single largest impact in the oncologic aspect of our specialty. While it has been repeatedly shown that local recurrence rates have plummeted from unacceptably high double-digit to low single-digit rates even without neoadjuvant therapy [4], it has also been shown that the laparoscopic and minimally invasive management of rectal carcinoma seems at least equivalent to and perhaps in many aspects, better than TME by standard laparotomy [5, 6]. While the subjective improvement such as superior visualization and therefore magnification are harder to quantify, the objective ones such as improved lymph node yield, shorter hospital stay, reduced pain, and expedited resolution of ileus have all been repeatedly proven.

S. D. Wexner $(\square) \cdot$ M. Berho

Department of Pathology, Cleveland Clinic Florida,

Weston, FL, USA

e-mail: wexners@ccf.org; kalasks@ccf.org
Whether the TME is performed by laparotomy or by laparoscopy, it has shown us that it is not the distal but rather the radial margin and the complete and intact nature of the excised mesorectum that are the predictors of local recurrence [7]. Quality indicators for these metrics are in the hands and eyes of our pathologists.

The role of the pathologist in the treatment of rectal cancer has dramatically evolved in part due to the emergence of the multidisciplinary team concept. Several studies have shown an improvement in outcomes in cases where treatment strategies are based on the consensus of opinions from several specialties [8]. The gross and microscopic analysis of the rectal cancer specimen is crucial not only to predict prognosis but also to tailor therapy for each individual patient. Several simple, concrete, and easy-to-evaluate parameters including the plane of surgery at which the dissection took place, the distance of the tumor to the resection margins, and the number of lymph nodes dissected should always be incorporated into the pathology report.

The plane of surgery or mesorectal integrity has been demonstrated to be one of the most important factors of both local and systemic cancer relapse [9]. According to where the dissection took place, the plane of surgery in rectal cancer surgery can be divided in three categories:

Mesorectal plane The rectum is dissected from the pelvis en bloc with the mesorectum and the totality of its lymph nodes. This surgery results in a complete bulky mesorectum with only minor irregularities.

Intramesorectal plane The mesorectum is disrupted during the dissection and irregularities can be seen on its surface without exposure of the muscularis propria.

Muscularis propria plane The muscularis propria is exposed, and the circumferential margin of resection is at 
the muscle layer increasing the possibilities of encountering a positive margin.

As previously mentioned, in the past decades, the emphasis has shifted from the distal to the circumferential margin of resection as the result of several large studies that have proven that the status of the radial or circumferential margin of resection is of paramount relevance to predict local recurrence [7].

Finally, despite great progress in understanding the molecular biology of colorectal cancer, the status of the lymph nodes remains the most important prognostic factor in colorectal cancer even after neoadjuvant chemoradiation [10]. Although a decrease in the number of dissected lymph nodes is expected for those patients who have received preoperative therapy, a few studies have shown that the larger the number of nodes found, the greater the possibility of discovering metastatic disease [11]. The surgeon's skills have the greatest influence as regards the integrity of the mesorectum and the status of the circumferential margin of resection, but pathologists have an equal, if not greater, responsibility in insuring an adequate lymph node harvest.

Although laparoscopic TME offers well-demonstrated advantages in clinical outcomes compared to open surgery, the impact of laparoscopy on pathological outcomes has been less consistently reported. Nevertheless, pathological parameters do not appear to be adversely affected by this technique [6], and the influence of the TAMIS (transanal minimally invasive surgery) procedure on the quality of the mesorectum and status of the circumferential margin of resection remains to be proven.

The huge global impact of laparoscopy left both manufacturers and innovators with a thirst for another similar leap forward. De Lacy et al. proposed the concept of natural orifice transanal endoscopic surgery (NOTES) to allow a scarless minimally invasive procedure [12]. While the first known NOTES procedure was a transgastric cholecystectomy, other types of procedures evolved including transvaginal colectomy [13]. Perhaps the most widespread use of NOTES is actually NOSE (natural orifice specimen extraction), a technique that has been described by many surgeons around the world using a myriad of slightly varied monikers [14]. We have shown that distal rectal adenocarcinomas can be treated with laparoscopic restorative proctectomy including TME with either mucosectomy or intersphincteric dissection and then NOSE including transperineal colonic J-pouch construction and hand-sewn or stapled coloanal anastomosis [15]. This hybrid NOTES technique has significant appeal since all abdominal incisions other than the port sites and the stoma site can be avoided. The natural step forward seems to be a transanal mesorectal excision. Sylla et al. [16] showed the feasibility of a hybrid abdominal and transanal approach using transanal endoscopic microsurgery (TEM) in five patients. Subsequently, Atallah et al. and Antonio Lacy et al. described the hybrid technique of TME using a transanal port $[12,17,18]$. In this issue, Atallah et al. present the results of their first 20 patients with rectal cancer who underwent transanal TME with a median follow-up of 6 months [12]. In both the Atallah and the de Lacy series of TAMIS-TME, the minimum threshold of 12 nodes was achieved in $80 \%$ of patients $[12,18]$. To assess mesorectal integrity Atallah et al. use their own modification of the method described by Quirke [8] (grade 1 defects of the mesorectal fascia $\geq 5 \mathrm{~mm}$; grade 2 defects of the mesorectal fascia of $<5 \mathrm{~mm}$; grade 3 intact mesorectum). In comparison, no details of the method were given by de Lacy et al. [12] who merely reported that "the quality of the mesorectal plane was reported as satisfactory in all the specimens." The classification used by Atallah is both unclear and unfortunately not validated; although their grade 2 and 3 TMEs correspond to the Quirke's well accepted "mesorectal plane," the clinical significance of the new classification is unknown. Thus, despite their report that the "mesorectal plane" was achieved in 17 of the 19 patients in whom the mesorectum was assessed (89 \%), the results may not be completely compatible. While I applaud these very technically gifted and academically respected authors for their honesty, I am quite concerned that we should be advocating this platform. While the authors note that the procedure can be successfully used for treatment with curative intent of patients with rectal cancer, they have unfortunately fallen rather short of proving this hypothesis. Given the development that we have seen during the last three decades, it would be tragic to lose the ground gained merely to allow a slight cosmetic enhancement by avoiding an abdominal incision. Although the technique may well hold promise for the future, it is clearly not yet "ready for prime time" and requires more diligent development by experts such as Dr. Larach and his group. The appeal and the theoretical advantage of this procedure will undoubtedly provide the impetus for these thought leaders and others including Lacy, Sylla, and Rattner bring this technology forward for more widespread use.

Conflict of interest Dr. Wexner is a paid consultant and receives royalty/inventor's income from intellectual property licensed to Karl Storz Endoscopy.

\section{References}

1. Heald RJ, Ryall RD (1986) Recurrence and survival after total mesorectal excision for rectal cancer. Lancet 1:1479-1482

2. Nigro ND, Vaitkevicius VK, Buroker T, Bradley GT, Considine B (1981) Combined therapy for cancer of the anal canal. Dis Colon Rectum 24:73-75 
3. Habr-Gama A, Sabbaga J, Gama-Rodrigues J et al (2013) Watch and wait approach following extended neoadjuvant chemoradiation for distal rectal cancer: are we getting closer to anal cancer management? Dis Colon Rectum 56:1109-1117

4. Wexner SD, Rotholtz NA (2000) Surgeon influenced variables in resectional rectal cancer surgery. Dis Colon Rectum 43:1606-1627

5. Fleshman JW, Wexner SD, Anvari M et al (1999) Laparoscopic vs. open abdominoperineal resection for cancer. Dis Colon Rectum 42:930-939

6. Boutros M, Hippalgaonkar N, Silva E, Allende D, Wexner SD, Berho M (2013) Laparoscopic resection of rectal cancer results in higher lymph node yield and better short-term outcomes than open surgery: a large single-center comparative study. Dis Colon Rectum 56:679-688

7. Nagtegaal ID, Quirke P (2008) What is the role for the circumferential margin in the modern treatment of rectal cancer? J Clin Oncol 26:303-312

8. Brown G (2012) Specialist multidisciplinary team working in the treatment of cancer. BMJ 26:344

9. Quirke P, Steele R, Monson J et al (2009) Effect of the plane of surgery achieved on local recurrence in patients with operable rectal cancer: a prospective study using data from the MRC CR07 and NCIC-CTG CO16 randomised clinical trial. MRC CR07/ NCIC-CTG CO16 Trial Investigators; NCRI Colorectal Cancer Study Group. Lancet 7:373

10. Klos CL, Shellito PC, Rattner DW et al (2010) The effect of neoadjuvant chemoradiation therapy on the prognostic value of lymph nodes after rectal cancer surgery. Am J Surg 200:440-445

11. Wang H, Safar B, Wexner SD, Denoya P, Berho M (2009) The clinical significance of fat clearance lymph node harvest for invasive rectal adenocarcinoma following neoadjuvant therapy. Dis Colon Rectum 52:1767-1773

12. de Lacy AM, Rattner DW, Adelsdorfer C et al (2013) Transanal natural orifice transluminal endoscopic surgery (NOTES) rectal resection: "down-to-up" total mesorectal excision (TME) short-term outcomes in the first 20 cases. Surg Endosc 27:3165-3172

13. Liu L, Chiu PW, Reddy N et al (2013) APNOTES Working Group. Natural orifice transluminal endoscopic surgery (NOTES) for clinical management of intra-abdominal diseases. Dig Endosc 25:565-577

14. Wexner SD, Edden Y (2009) NOTES/NOSE/NOSCAR/LATAS: what does it all mean? Tech Coloproctol 13:1-3

15. Takano S, Boutros M, Wexner SD (2014) Laparoscopic restorative proctocolectomy with ileal J-pouch creation through the umbilical port. J Am Coll Surg 218:e5

16. Sylla P, Bordeianou LG, Berger D et al (2013) A pilot study of natural orifice transanal endoscopic total mesorectal excision with laparoscopic assistance for rectal cancer. Surg Endosc 27:3396-3405

17. Atallah S, Albert M, DeBeche-Adams T, Nassif G, Polavarapu H, Larach S (2013) Transanal minimally invasive surgery for total mesorectal excision (TAMIS-TME): a stepwise description of the surgical technique with video demonstration. Tech Coloproctol $17: 321-325$

18. Atallah S, Martin-Perez B, Albert M (2013) Transanal minimally invasive surgery for total mesorectal excision (TAMIS-TME): results and experience with the first 20 patients undergoing curative-intent rectal cancer surgery at a single institution. Tech Coloproctol. doi:10.1007/s10151-013-1095-7 